\title{
A Novel Infrared (IR) Based Sensor System for Human Presence Detection in Targeted Locations
}

\author{
Dr. Kakelli Anil Kumar \\ Associate Professor, SCOPE, Vellore Institute of Technology, Vellore, TN, 632014 \\ E-mail: anilsekumar@gmail.com \\ Omkar Dhadge \\ SCOPE, Vellore Institute of Technology, Vellore, TN, 632014 \\ E-mail: ovddhadge@gmail.com
}

Received: 19 September 2018; Accepted: 16 October 2018; Published: 08 December 2018

\begin{abstract}
Human presence detection is a continuously sought of an issue by the scientific community. Visual camera-based technologies have emerged recently with low cost and easy usage. However, these technologies have been increased the user privacy issues. Hence it is highly essential to design a human detection system without compromising the user privacy, comfort, cost and easy deployment. The pyroelectric infrared (PIR) based sensor systems are introduced however this technology is incapable to detect the presence of stationary human because it can detect the fluctuating signals only. In this paper, we have proposed a novel infrared (IR) based sensor system to detect the human presence either mobile or immobile in targeted locations with high accuracy. The proposed infrared (IR) sensor is designed to sense the heat radiation emitted by the human body, it detects the human presence accurately in targeted locations. The proposed IR based sensor system has successfully deployed in a targeted location and tested successfully for detecting the human presence and also other objects.
\end{abstract}

Index Terms - Human Presence, IR sensor, PIR sensor, body temperature, target location.

\section{INTRODUCTION}

Automation of devices has revolutionized the comfort levels of the human life. New devices are equipped with automated systems for reducing the need for human effort. The eco-friendly automation is relying on sensors, transducers, and control systems. Therefore researchers have focused much on developing sensors with new technologies for sensing and detection of the targeted objects. Human exhibits various unique characteristic like body temperature, shape and color, and release of $\mathrm{CO}_{2}[1]$

Our research work aims to create a system which can respond to the human body temperature for detection. IR sensors are best and accurate to measure radiated heat by human body. It is the most precise characteristic for human detection in sensitive locations. It is well known that anything that has a temperature above absolute zero emits infrared radiation [2]. Another crucial challenge is human body temperature is not constant which varies from 36.5 to $37.5^{\circ} \mathrm{C}$ due to wearable clothes, external conditions like seasons and day and night timings. The primary factors for localization of human presence may include the distance between the heat source and sensor system [3]. The body surface radiation is absorbed by the sensor can range from $20^{\circ} \mathrm{C}$ to $40^{\circ} \mathrm{C}$ depending on the external conditions. For indoor applications the sensor should be able to differentiate human from static objects/things like metal objects, wooden furniture and plastic objects even though the human is mobile or immobile. The parameters like distance, angle and coordinates are calculated using the position of the sensing device for accurate human location in the field of view (FOV) or targeted location. Therefore, it is highly essential to choose the IR sensors for concerning range, accuracy, and FOV. This research work has proposed Omron D6T-44L-06 MEMS IR sensor which results 4x4 array pixel low-resolution thermal image as graphical output. The outcome of this work is to visualize the thermal signature of human and human-object interaction in two dimension (2D) using thermopile modules of Omron D6T sensor. The position of sensors is also another crucial parameter to consider for better coverage of FOV. The number of required sensors is based on size of FOV and human count by avoid the interference range between multiple sensors [4].

Unlike CCTV cameras, the low resolution 2D thermal signature output of the thermopile sensor is not smooth to interpret thereby reduces the privacy concerns of the users. The 2D array matrix output of Omron D6T MEMS series sensor identifies the pixels of relatively high temperature than the surrounding and gives the location and presence of the human in range [5]. The PIR sensors were most commonly preferred to detect human presence before the thermal sensors due to low cost. However PIR sensor can able to identify the signals from the person in motion and simultaneously it results the false negative when the person was stationary. PIR sensor also has another limitation of time transmission delay of approximately 2 seconds to 9 minutes. On the other hand, the thermal sensor can continuously differentiate a stationary human from other static or dynamic objects. 
The thermal sensor is more capable device for human detection than existing technologies like visual camera. Although visual camera gives the high quality images higher than $4 \times 4$ pixel sensing array, whereas using visual camera at night time required continuous illumination of light [6]. The characteristic of the thermopile does not allow energy emitting to the surrounding which keeps the sensor unaffected. Hence, visual camera and thermopile sensor based technologies are failed to achieve human privacy and accuracy [7].

\section{APPliCATIONS OF THE PROPOSED System}

The thermal sensory system is a new field has many revolutionary capabilities.

\section{A. Security and Privacy}

The human detection systems are very important in security and surveillance fields. Due to the limitations of PIR sensor, now the emerging applications are demanding thermal sensor based human/object detection systems. To further improve the security aspects, thermal sensors can be coupled with PIR and ultrasonic sensor to make the device more perfect for detecting each human activity [8].

\section{B. Disaster Relief}

Every year thousands of people die in natural disasters, a significant percentage of them can be saved if efficient detection devices are available. Therefore, smart thermal sensing devices can help to detect the humans buried under the rubble. Currently, these thermal sensors are not able to penetrate deeply into the rubble, but they still can provide an extra 3-8m arm to the rescuers which indeed a significant role for saving the people [9].

\section{Medical Treatments and Safety from Radiation}

Thermal imaging plays a key role in medical treatments. If adequately calibrated sensors can detect the variations found in thermal signature of the patients which helps to detect the appropriate problem and diagnosis [10]. One of the most recent utilization of these proposed sensors involves alert the people from radiation exposure.

\section{Energy Management Systems}

The automated devices can use the thermal sensors like Omron D6T to detect the presence of the human to control home appliances. Smart thermostats have developed to adjust the indoor temperature automatically and regulate the room environment through the mobile applications [11].

\section{E. Activity Recognition and Localization}

Another critical application of Omron D6T thermal sensor deals with assisting older and physically challenged people. The sensor was successfully able to provide positive results in detecting human activities like sitting, lying, falling, and many more. These sensors can also connect to the home network which can make home and human-activity monitoring by these category people.

\section{F. Advanced driver assistance system (ADAS)}

ADAS involves heterogeneous sensors which are installed in vehicle to assist the drivers while driving. The sensory system accurately alerted the driver about pedestrian near the vehicle through IR sensor.

\section{G. Thermal Cameras and Fire Safety}

Well-equipped hardware components and image processing algorithms results the quality thermal images. A thermal camera provides night vision which most of the visual cameras lack and therefore thermal camera is much more suitable for alarm.

\section{OMRON D6T-44L-06 MEMS THERMAL SENSOR}

The proposed OMRON D6T sensor has three components: i) silicon lens cap ii) MEMs thermopile module iii) circuits for converting analog data into the digital values. The role of silicon lens is to absorb the farinfrared rays emitted by the target object using the thermopile sensor. The electromotive force develops between the two nodes of the thermopile because of the difference in temperature which is used by the analog circuit to give output digital temperature value. This calculated temperature is output through an I2C communication bus as mentioned in Table 1. OMRON's D6T-441-06 thermal sensor is a non-contact infra-red sensor and has a high sensitivity that enables detection of stationary human presence in a room without the need for any movements. It can also locate and estimate the number of people in a room and recognize if a person is leaving a predefined area. Additionally, Omron D6T sensors will give a continuous output of the temperature values detected without any delay [12].

\section{A. System Design and Result Analysis}

The hardware components of the proposed sensor are Omron D6T-44L-06 MEMs thermal sensor, microcontroller (Arduino Uno), jumper wires and few pull up resistors. For programming, the open source Arduino IDE is used and program was uploaded to microcontroller and processing IDE to display the $4 \mathrm{X} 4$ pixel matrix as graphical output. IR sensor is highly sensitive, proper connections are highly necessary with the controller. Datasheets are available on the sensor-web are used to set up the system [13].

A thermopile Omron D6T thermal sensor comprises of various thermocouples associated with arrangement. A thermocouple produces a voltage when transmitter (i.e., metal) acquires contact with the heat. This voltage creating impact is known as Seebeck impact. A thermocouple sustains Seebeck impact through hot and cold intersections. At the cold intersection, the temperature is same as encompassing temperature since silicon is exceedingly conductive. At the heated intersection, the temperature is reliant on the stream speed. The voltage generated between the hot and cold intersections of the thermopile help to calculate the 
temperature values which will be sent from the sensor to the microcontroller using equation 1.

$$
V=(S a-S b) *(T h-T c)
$$

Where $\mathrm{Sa}$ and $\mathrm{Sb}$ are the Seebeck coefficients of the two materials and Th and Tc are the temperatures of the hot and cold junctions respectively.

Table 1. Characteristics of OMRON D6T-44L-06 Sensor




From equation (1), the created voltage has relied upon the immediate temperatures. The output of a thermopile is independent of the state path of the system and only depends on the current state. This sensor is appropriate to distinguish the condition of a framework even in a constant state [14]. The human presence detecting range using thermal sensor varies from 3-5 meters from its calibrated position, but the temperature values vary over the distance between the sensor location and the human. The temperature falls as the distance between human and sensor increases even though the FOV area gets more prominent the occupied area by target reduces thus allowing background temperature to prevail and affect the detection rate. Similarly, the detection temperature increases with the closeness of the object from the sensor. To reduce the noise levels as much as possible, the difference between the maximum and minimum temperature detected has to be greater than $2^{\circ} \mathrm{C}$. It is also important that the background temperature can affect the detection accuracy of the object if the object is smaller than the sensitivity area. Such as when the surrounding temperature is nearly equal to the body temperature of the human, it becomes a tricky situation to detect humans since the difference between the brightness of pixels on the sensing array will be negligible $[15,16]$.
In Figure 1, it can be observed the connection required between the sensor and the microcontroller. To view the temperature values in the serial monitor of Arduino IDE. The serial monitor displays the recorded temperature output of the sensor as shown in figure 2. After successfully receive the temperature data from the proposed sensor and processed these values in processing IDE. Processing IDE is capable of reading the data from serial ports and output the information using the graphical display. The Arduino IDE and processing IDE are capable to access the same serial port but not simultaneously because it will result an error due to port busy. It visualized the $4 \times 4$ pixel array with pixels color coded as desired for a specific range of temperature. The pixels with the specific color having the range from $29^{\circ} \mathrm{C}$ to $38^{\circ} \mathrm{C}$ which are likely to describe the human presence. Finally to detect the probability of the person identification, it is important to check the temperature of brighter pixels and estimated the deviation with other pixels. The higher variance results more likely is the chance of human presence and located in the direction of the brightest pixel as shown in figure 2. Now, following are the results of few test cases conducted in our experimentations.

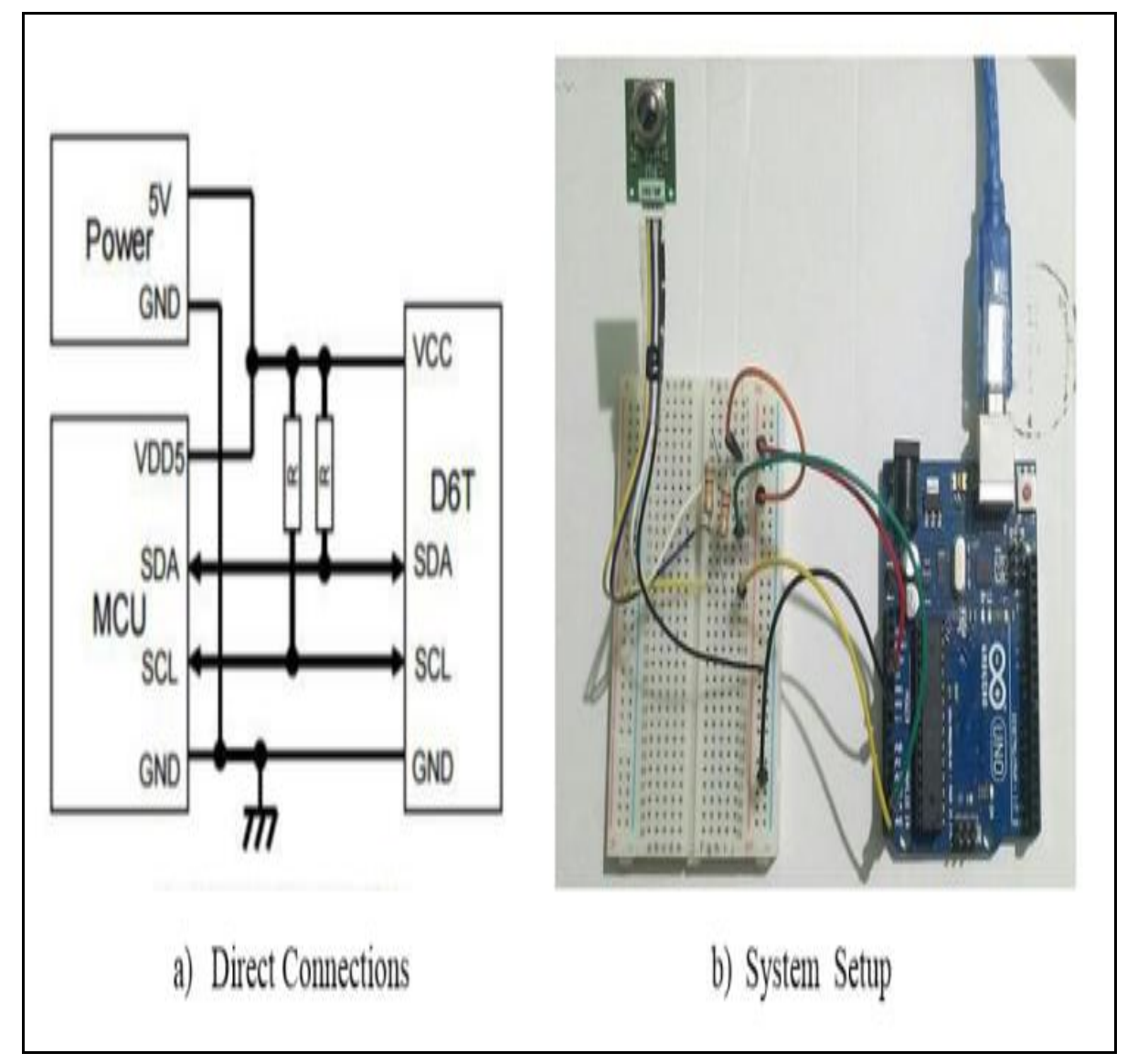

Fig.1. Logical Connections of OMRON D6T Sensor and Arduino MC 


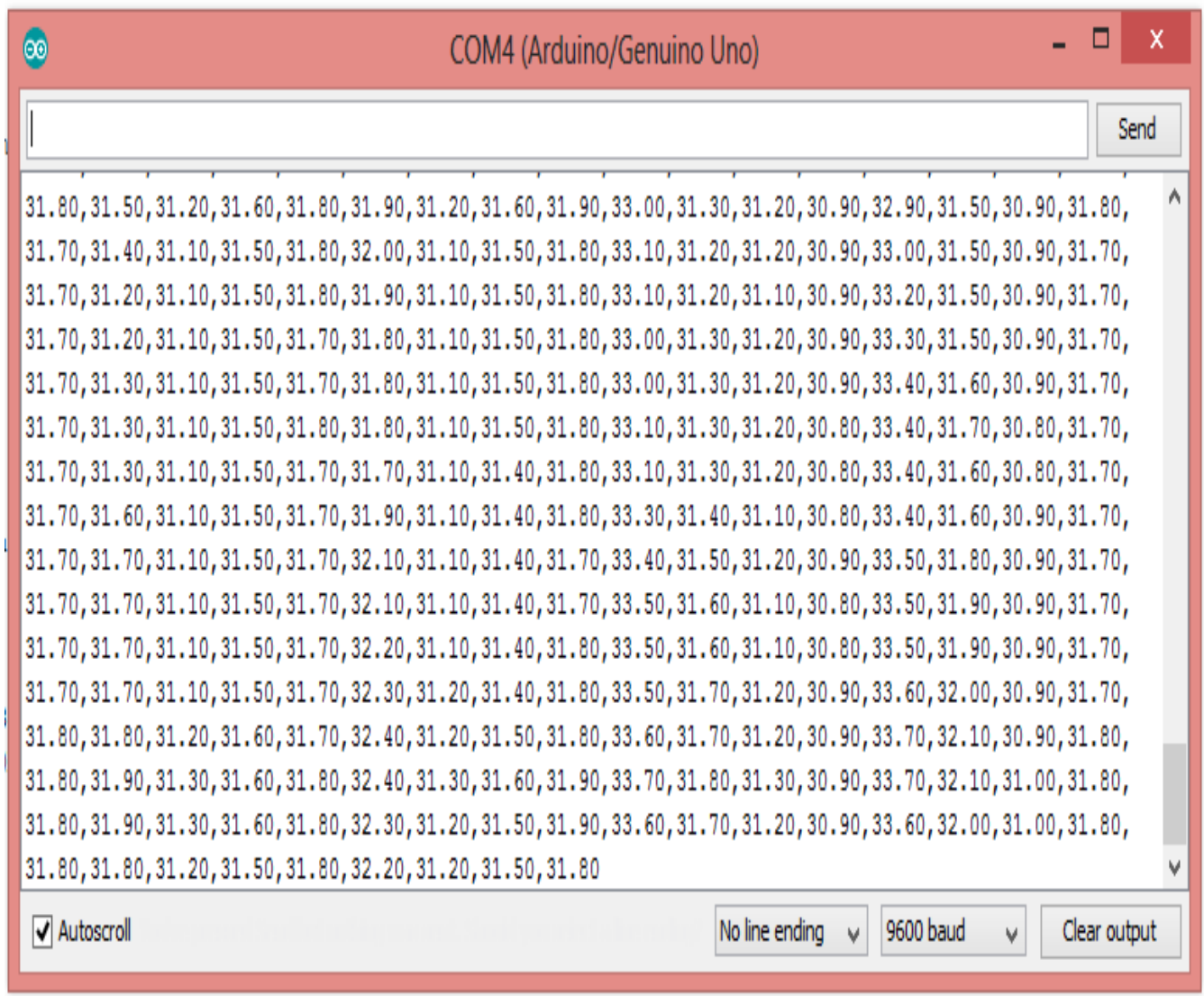

Fig.2. The Output Temperature Values in Serial Monitor of Arduino

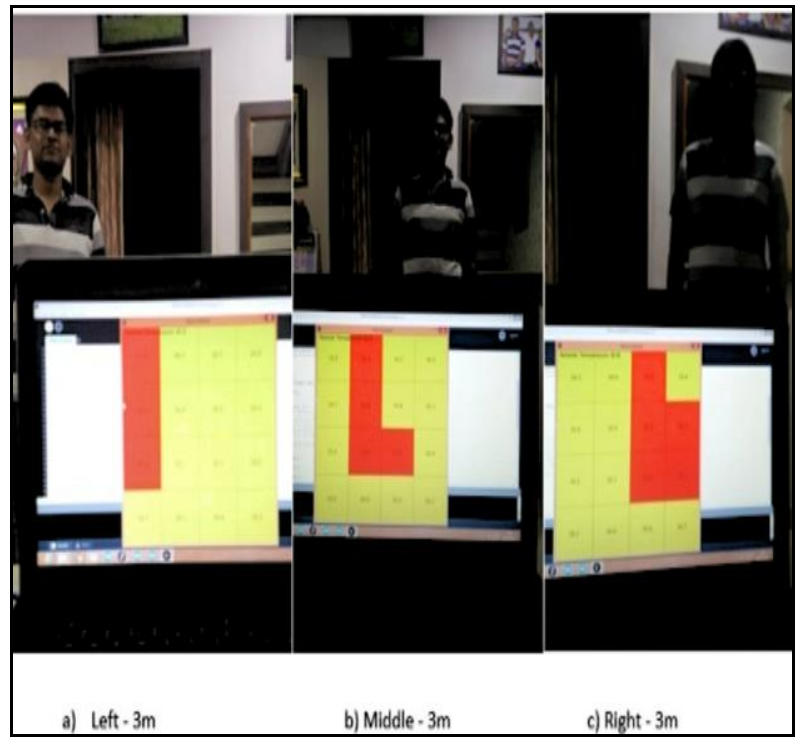

Fig.3. Graphical Output of OMRON D6T Sensor of $3 \mathrm{~m}$ Distance

\section{B. $\quad$ Test Case 1 with 3 Meter Distance}

Figure 3 shows the graphical output of first experimentation with the range of 3 meters distance between human and sensor device. The graphical output of $4 \times 4$ pixel array is used to determine the location and presence of the human. In test case 1, the red pixels denote the location of the human detected in the FOV of the sensor. The red represents the human-pixels, and another color represents non-human pixels. The temperature readings are displayed over the pixel to see the difference between the body and background temperature. It is also clearly observed that the proposed sensor can be used for both detection and localization of humans as it identifies the left, middle and right position of the target. Human presence is detected in red pixels and room temperature in yellow pixels. Human presence detected using our sensor at three different locations like left, center and right as shown in figure $3 \mathrm{a}, 3 \mathrm{~b}$ and $3 \mathrm{c}$.

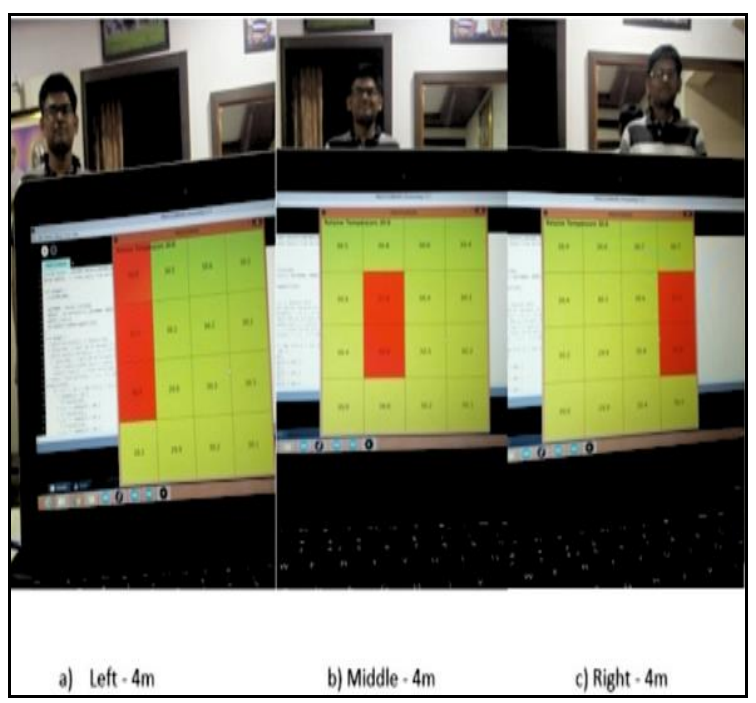

Fig.4. Graphical Output of OMRON D6T Sensor of $4 \mathrm{~m}$ Distance 


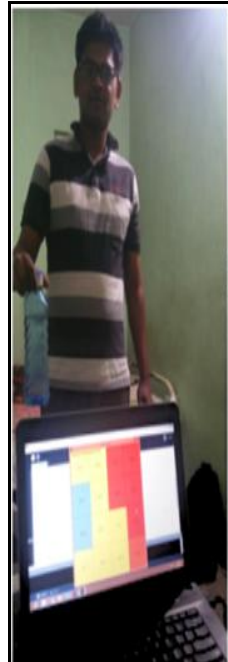

(a)

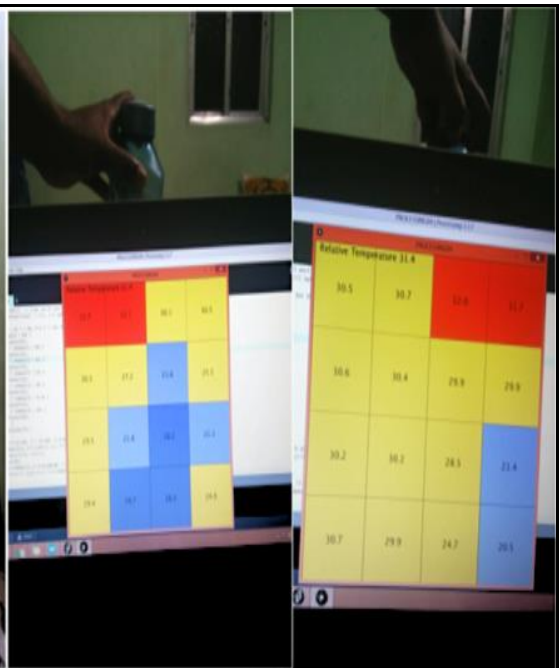

(b) (c)
Fig.5. Graphical Output of OMRON D6T Sensor for Detecting Human and Cold Water

\section{C. $\quad$ Test case 2 with 4 Meter Distance}

The only difference in test case 1 and test case 2 is the distance between the human and sensor. The second experiment has given less count of red pixels compared to test case 1 as shown in figures 4 . It is detected that human body temperature falls with respect to increase the distance between human and sensor.

\section{Test Case 3 with 3 Meter Distance and other Object}

Test Case 3 shows that the sensor is capable of detecting the surface temperature of both living and nonliving objects. The red pixels which range from $31^{\circ} \mathrm{C}$ to $33{ }^{\circ} \mathrm{C}$ represents the surface temperature of the human body whereas the blue pixels represents the surface temperature of cold water in a bottle of temperature range $20{ }^{\circ} \mathrm{C}$ to $24{ }^{\circ} \mathrm{C}$. Graphical output in figure 5 shows, cold water in blue pixels, human presence in red pixels and room temperature in yellow pixels. From figures $5 \mathrm{a}, 5 \mathrm{~b}$ and $5 \mathrm{c}$, it is observed that human presence at right side and cold water at left side, cold water at center lower and human presence at left upper, and cold water at right lower and human at right upper respectively. The presence of human is detected efficiently by separating the human-pixels from non-human pixels with different color coding of pixels from figure 5 .

\section{CONCLUSION AND FUTURE WORK}

The proposed sensor system is convenient to use and deploy for human detection applications. The proposed human detection sensor is comparatively cheap and better optimal range, high accuracy and better field of view (FOV). The installation of the sensor at the proper position may increase the better coverage of the targeted region. The $4 \times 4$ pixel array output is provided quality information, and monitored the desired activities in FOV. The performance evaluation of the proposed system has proven that it is high-quality, secure and energy efficient.
The proposed system is quite useful for essential applications like security, fire-safety, disaster relief and smart household appliances. As a future work, we are working to introduce an improved sensing system which can separate the human surface temperature (humanpixels) from surrounding temperature (non-human pixels) with high accuracy. The improvements in the proposed sensor system are introducing like Fresnel external lens on the sensor to get a brighter images.

\section{REFERENCES}

[1] A. D. Alzughaibi, H. A. Hakami, and Z. Chaczko., Review of Human Motion Detection based on Background Subtraction Techniques. International Journal of Computer Applications, 2015, 122(13):975-8887.

[2] S. Narayana, R. V. Prasad, V. S. Rao, T. V. Prabhakar, S. S. Kowshik, and M. S. Iyer., PIR sensors: characterization and novel localization technique. 14th Int. Conf. on Information Processing in Sensor Networks, 2015, 142153.

[3] K. Lee, C. Y. Choo, H. Q. See, Z. J. Tan, and Y. Lee., Human detection using Histogram of Oriented Gradients and human body ratio estimation. Proceedings of 3rd IEEE International Conference on Computer Science and Information Technology, 2010, 4: 18-22.

[4] M. Hein, R. Stöber, M. Meiler, D. Schaller, R. Zehle, G. Fischerauer, J. Bauer, J. Bürner, T. Becher, M. Feller, and J. Maul., Sensor solutions for an energy-efficient and user-centered heating system. Journal of Sensors and Sensor Systems, 2017, 6(1):27-35.

[5] R. Bogue., Recent developments in MEMS sensors: a review of applications, markets and technologies. Sensor Review, 2013, 33(4):300-304.

[6] M. Popescu, B. Hotrabhavananda, M. Moore, and M. Skubic., VAMPIR - An Automatic Fall Detection System Using a Vertical PIR Sensor Array. Proceedings of the 6th International Conference on Pervasive Computing Technologies for Healthcare, 2015.

[7] X. Jin, S. Member, S. Sarkar, A. Ray, and S. Gupta., Target Detection and Classification Using Seismic and PIR Sensors. IEEE Sensors Journal, 2012, 12(6):17091718.

[8] L. Wang., Human infrared signal recognition using single PIR detector. Proceedings of 4th International Congress on Image and Signal Processing, 2011, 5:2664-2668.

[9] A. Gupta and B. Thomas., A New Revolutionary Infrared Life Detection System Using ATmega168. International Journal of Embedded Systems and Applications, 2012, 2(3):117-122.

[10] C. Deutsch, R. Krger, K. Saito, S. Yamashita, Y. Sawanoi, B. Beime, and P. Bramlage., Comparison of the Omron RS6 wrist blood pressure monitor with the positioning sensor on or off with a standard mercury sphygmomanometer. Blood Pressure Monitoring, 2014, 19(5):306-313.

[11] S. Parnin and M. M. Rahman., Human Location Detection System Using Micro-Electromechanical Sensor for Intelligent Fan. IOP Conference Series: Materials Science and Engineering, 2017, 184:1.

[12] A. A. S. Mohammed, W. A. Moussa, and E. Lou., Highperformance piezoresistive MEMS strain sensor with low thermal sensitivity. Sensors, 2011, 11(2):1819-1846.

[13] S. Ferdoush and X. Li., Wireless sensor network system design using Raspberry Pi and Arduino for environmental monitoring applications. Procedia Computer Science, 
2014, 34:103-110.

[14] J. T. W. Kuo, L. Yu, and E. Meng., Micromachined thermal flow sensors-A review Micromachines, 2012, 3(3):550-573.

[15] A. A. Chandra, Y. Lee, B. M. Kim, S. Y. Maeng, S. H. Park, and S. R. Lee., Review on sensor cloud and its integration with arduino based sensor network. International Conference on IT Convergence and Security, 2013.

[16] F. Erden and A. Çetin., Hand gesture based remote control system using infrared sensors and a camera. IEEE Transactions on Consumer Electronics, 2014, 60(4):675680.

\section{Authors' Profiles}



Dr. Kakelli Anil Kumar, born in 1982. He received his $\mathrm{Ph}$. D degree in Computer Science and Engineering from Jawaharlal Nehru Technological University Hyderabad, TS, India and currently working as Associate Professor in School of Computer Science and Engineering (SCOPE), Vellore
Institute of Technology, Vellore, Tamil Nadu, India. His main research interests include wireless sensor network, mobile ad_hoc networks, cloud computing and information security.

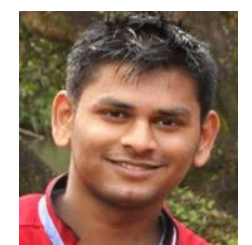

Mr. Omkar Dhadge, born in 1996. He received his bachelor degree in Computer Science and Engineering with the specialization of bioinformatics from School of Computer Science and Engineering (SCOPE), Vellore Institute of Technology, Vellore, TN, India. Currently he is pursuing Master of Science (MS) in Computer Science at NYU, USA. His main research interests include wireless sensor network, network security and networked control system.

How to cite this paper: Kakelli Anil Kumar, Omkar Dhadge,"A Novel Infrared (IR) Based Sensor System for Human Presence Detection in Targeted Locations", International Journal of Computer Network and Information Security(IJCNIS), Vol.10, No.12, pp.34-40, 2018.DOI: 10.5815/ijcnis.2018.12.04 